hep-th/9606017

RU-96-46

\title{
IR Dynamics on Branes and Space-Time Geometry
}

\author{
Nathan Seiberg \\ Department of Physics and Astronomy \\ Rutgers University \\ Piscataway, NJ 08855-0849 \\ seiberg@physics.rutgers.edu
}

\begin{abstract}
We consider the type I theory compactified on $T^{3}$. When the D5-brane wraps the $T^{3}$ it yields a D2-brane in seven dimensions. In the leading approximation the moduli space of vacua of the three dimensional field theory on the brane is $T^{4} / \mathbf{Z}_{2}$. The dual $\mathrm{M}$ theory description of this theory is a compactification on K3 and our 2-brane is the eleven dimensional 2-brane at a point in K3. We use this fact to conclude that strong coupling IR effects in the three dimensional theory on the brane turn its moduli space into a K3. This interpretation allows us to solve various strongly coupled gauge theories in three dimensions by identifying their Coulomb branch with a piece of a (sometime singular) K3.
\end{abstract}

May 1996 
D-branes [1] appear to be interesting probes of space-time geometry and background gauge fields [2-6]. In particular, motivated by observations of Sen [7] the authors of [5] showed how the background $\tau$ parameter of IIB compactifications known as $\mathrm{F}$ theory [8] can be probed. The relevant D-brane is the D3-brane of the type II theory, whose four dimensional world volume gauge theory has such a $\tau$ parameter. In the leading approximation the $\tau$ parameter is given by the tree level, short distance value in that four dimensional gauge theory. The exact value of $\tau$ in $\mathrm{F}$ theory is given by the long distance value in the gauge theory as determined in [9].

It is interesting that the values of the space time background fields are given by the long distance parameters of the theory on the brane rather than by their short distance, tree level values. Since the brane is only a probe of the space time fields, this observation raises an interesting question. How do these background fields adjust themselves to be compatible with the long distance dynamics on the probes? Are they affected by the probes or is it merely that their values are consistent with any probes which can be used?

In [5] a compactification to eight dimensions was discussed. Here we will study lower dimensional examples.

Consider the type I theory compactified on $T^{3}$ with generic $S O(32)$ Wilson lines. Our probe will be a D5-brane which wraps the compact $T^{3}$ yielding a 2-brane in seven dimensions. The collective coordinates of the brane are an $S U(2)$ gauge theory with some "quarks" [10]. The moduli space of vacua of the theory on the brane in the leading approximation is parametrized by $S U(2)$ Wilson lines. This space is $\mathcal{M}_{c l}^{0}=T^{3} / \mathbf{Z}_{2}$. At the singularities of $\mathcal{M}_{c l}^{0}$ the $S U(2)$ gauge symmetry is restored. At generic points on $\mathcal{M}_{c l}^{0}$ the $S U(2)$ gauge symmetry is Higgsed to $U(1)$. The photon in the low energy three dimensional theory can be dualized to another massless boson which extends the moduli space by adding a circle which is fibered over $T^{3} / \mathbf{Z}_{2}$. The fiber is singular over the singularities in the base.

The space-time $S O(32)$ Wilson lines appear as mass terms in the theory on the brane. Therefore, for generic values of these Wilson lines there are no massless quarks at the singularities of $\mathcal{M}_{c l}^{0}$. However, at various points on $\mathcal{M}_{c l}^{0}$ there are massless "electrons" (we distinguish them from quarks which couple to massless non-Abelian gauge fields) where the $S U(2)$ Wilson line cancels the $S O(32)$ Wilson line. At these points the duality transformation on the photon is complicated. However, at the classical level $\mathcal{M}_{c l}^{0}$ is still smooth there.

It can be helpful to perform a $\mathrm{T}$ duality transformation on all three compact coordinates of the compactification. The resulting type I' theory is the type IIA theory compactified on $T^{3}$ with 8 orientifolds, which are 6-branes and sixteen D6-branes which fill the non-compact dimensions. The probe is then the D2-brane of the IIA theory which 
is at a point in the compact space. The orientifold points correspond to the enhanced $S U(2)$ gauge symmetry on the brane and the D6-branes correspond to the points with massless electrons.

To summarize, the classical moduli space of vacua of the three dimensional theory on the brane, $\mathcal{M}_{c l}$, is a four dimensional space whose base is $\mathcal{M}_{c l}^{0}=T^{3} / \mathbf{Z}_{2}$ and whose fiber $S^{1}$ is singular over the 8 singularities of the base. The resulting classical moduli space is $\mathcal{M}_{c l}=T^{4} / \mathbf{Z}_{2}$.

What happens to $\mathcal{M}_{c l}$ in the quantum theory? By three dimensional supersymmetry it has to be a hyper-Kahler manifold which is approximately $T^{4} / \mathbf{Z}_{2}$. If all the singularities are smoothed out, it must become K3. If this is the case, our 2-brane which is embedded in seven dimensions is at a point in K3. This is precisely the picture implied by compactifying $\mathrm{M}$ theory on K3 with the eleven dimensional 2-brane identified with our 2-brane. As a consistency check of this proposal, note that in eleven dimensions the 2-brane is dual to the 5 -brane. Since it is at a point on the compact K3, its dual in seven dimensions is a string obtained by wrapping the eleven dimensional 5-brane on K3. According to the conjectured $\mathrm{M}$ on K3/heterotic on $T^{3}$ duality [11, this object is the heterotic string [12. Therefore, our 2-brane is dual to the heterotic string. Indeed, it started its life as the 5-brane of the type I theory which is dual to the heterotic string.

Instead of trying to prove that the moduli space becomes $\mathrm{K} 3$, we will assume that this is the case. As we said, this assumption follows from the conjectured $\mathrm{M}$ on K3/heterotic on $T^{3}$ duality.

We will now use this assumption as a powerful tool for studying the dynamics of three dimensional gauge theories with $N=4$ supersymmetry. The minimal such theory is obtained by considering a limit of the theory on the brane without higher dimension operators. This is the case when the dimensionful gauge coupling $g$ in the three dimensional

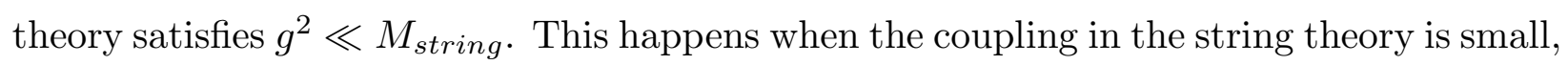
or equivalently, when the base $M_{c l}^{0}$ is much bigger than the fiber. Then we can study the neighborhood of various points in $M_{c l}^{0}$ for various values of the moduli.

Our three dimensional theories have $N=4$ supersymmetry. This algebra is generated by four real supercharges $Q_{\alpha}^{i}(i=1, \ldots, 4 ; \alpha=1,2)$ which are spinors of the Lorentz group $S O(1,2)$. Starting with a renormalizable theory with massless quarks, the Lagrangian has a global $\operatorname{Spin}(4)_{R} \sim S U(2)_{R 1} \times S U(2)_{R 2}$ symmetry under which the four supercharges form a vector. This symmetry includes the more familiar $S U(2)_{R} \times U(1)_{R}$ symmetry of the similar four dimensional theory with $N=2$ supersymmetry. The gauge fields are in vector multiplets. They include three scalars transforming as $(\mathbf{1}, \mathbf{3})$ under the global symmetry and a photon. Upon dualizing the photon, we get another scalar transforming 
as $(\mathbf{1}, \mathbf{1})$. Together with the other fields, this is a hypermultiplet. The scalars in ordinary hypermultiplets transform as $(\mathbf{2}, \mathbf{1}) \oplus(\mathbf{2}, \mathbf{1})$ or $(\mathbf{3} \oplus \mathbf{1}, \mathbf{1})$.

\section{$U(1)$ gauge theories}

The Coulomb branch of the tree level theory is $\mathbf{R}^{3} \times S^{1}$ where the $S^{1}$ factor parametrized by $\chi$ corresponds to the dual of the photon. The metric on this space is the obvious flat metric and the circumference of the circle is the gauge coupling $g$. Since the part of the metric, which involves the circle, $g^{2}(d \chi)^{2}$, is of order $g^{2}$, for consistency some higher order perturbative contributions of the same order should be included. For example, parameterizing $\mathbf{R}^{3}$ by polar coordinates $\vec{x}=(r, \theta, \phi)$ a one loop term $c \cos \theta d \phi \wedge F$ ( $c$ is a constant) can be generated. After the duality transformation the metric becomes $\frac{1}{g^{2}}(d \vec{x})^{2}+g^{2}(d \chi+c \cos \theta d \phi)^{2}$. Such a correction can change the asymptotic behavior of the space. The global Spin(4) symmetry is spontaneously broken on the moduli space away from the origin to $S U(2)_{R 1} \times U(1)$ since the scalars in the multiplet transform like $(\mathbf{1}, \mathbf{3} \oplus \mathbf{1})$. The broken $S U(2)_{R 2}$ factor acts on $\mathbf{R}^{3}$ in an obvious way and it is restored at the origin. The circle leads to another global $U(1)$ symmetry which is spontaneously broken. The quantum corrections could change this picture. In particular, in the example above the global $S U(2)_{R 2}$ acts also on the circle. Then, $S U(2)_{R 2}$ is completely broken and there is an unbroken global $U(1)$.

The theory without matter fields is free and the classical picture does not change in the quantum theory. However, with matter fields, electrons, the theory is interacting. The interaction is relevant at short distance and the theory is asymptotically free. Therefore, far out along the flat directions, where the electrons are heavy, the moduli space is given by the one loop corrected answer. Comparison with the theory on the brane can teach us about the quantum moduli space in the strongly coupled region.

$U(1)$ with one electron. This is the theory at generic values of the space time moduli (i.e. with generic K3) when in the type I' description the 2-brane is near one of the 16 D-branes. In the interior of the moduli space a singularity could develop associated with the massless electron. However, since our moduli space is part of a K3 at a generic point in its moduli space, which is smooth, the moduli space of the gauge theory cannot have singularities. We conclude that a $U(1)$ theory with one electron does not have a singularity in its moduli space and correspondingly, the fundamental electron is never massless.

$U(1)$ with $N_{f}>1$ electrons. In the type I' description this theory describes the situation when $N_{f}$ 6-branes touch each other and the 2-brane probe is near that point. The theory on the brane has an $S U\left(N_{f}\right)$ global symmetry while the space time theory has an $S U\left(N_{f}\right)$ gauge symmetry. Then, at the origin of the moduli space of the theory on 
the brane there are $N_{f}$ massless electrons. Out of that point there is a Higgs branch. As explained in [9], on that branch the $S U\left(N_{f}\right)$ global symmetry is broken to

$$
S U\left(N_{f}\right) \rightarrow \begin{cases} & \text { for } N_{f}=2 \\ U(1) & \text { for } N_{f}=3 \\ S U\left(N_{f}-2\right) \times U(1) & \text { for } N_{f} \geq 4\end{cases}
$$

The light hypermultiplets on the moduli space transform like

$$
\begin{array}{cc}
\mathbf{1} & \text { for } N_{f}=2 \\
\mathbf{1}_{1} \oplus \mathbf{1}_{0} & \text { for } N_{f}=3 \\
\left(\mathbf{N}_{\mathbf{f}}-\mathbf{2}\right)_{1} \oplus \mathbf{1}_{0} & \text { for } N_{f} \geq 4 .
\end{array}
$$

As in [13] we interpret this Higgs branch as the moduli space of an instanton in the space time theory. As a consistency check, note that (1) and (2) are consistent with that interpretation. In the quantum theory the Coulomb branch could be modified. As explained above, far out along the flat directions the moduli space is given by the one loop corrected answer. Unlike the previous cases, the singularity on the Coulomb branch must survive. This follows from the existence of a Higgs branch which goes out of it. A more precise characterization of the singularity comes from comparing it with the exact answer, which must be part of a K3. Here we are at a non-generic K3. The enhanced $S U\left(N_{f}\right)$ gauge symmetry in space time is associated with an $A_{N_{f-1}}$ singularity in K3. Therefore, the moduli space must have an $A_{N_{f}-1}$ singularity at the origin. Since the short distance theory is asymptotically free, we expect to have a different theory at long distance. There is no free field theory other than the original one which leads to the Higgs branch (11), (2). Therefore, the theory at the singularity is at a non-trivial fixed point of the renormalization group.

\section{$S U(2)$ gauge theories}

These theories are always interacting and asymptotically free for any number of flavors. The Coulomb branch is four real dimensional. In the classical theory it is $\mathbf{R}^{3} \times S^{1} / \mathbf{Z}_{2}$ where the $\mathbf{Z}_{2}$ which originates from the Weyl subgroup of the $S U(2)$ gauge theory acts on both factors as reflection. The metric on this space (away from the singularities) is flat and the circumference of the $S^{1}$ factor is the gauge coupling $g$. Again, for consistency we should add some higher order perturbative corrections to the metric of the same order in $g$. As with $U(1)$ gauge theories, the global $S U(2)_{R 2}$ symmetry acts on $\mathbf{R}^{3}$ in an obvious way. It is restored at the origin of the $\mathbf{R}^{3}$. The perturbative quantum corrections can make $S U(2)_{R 2}$ act also on the circle. In this case $S U(2)_{R 2}$ is spontaneously broken to a trivial group. The global $U(1)$ symmetry which is associated with translations of the 
circle is explicitly broken by instantons (the configurations which lead to monopoles in the analogous four dimensional theory).

$S U(2)$ with no flavors. We find this theory at a generic point of the space time moduli space, when in the type I' description the 2-brane probe is near one of the the orientifolds - the origin of $\mathbf{R}^{3}$. Far from there the moduli space of the gauge theory asymptotes to the one loop corrected answer. From the map to K3, the moduli space should be part of a smooth K3 (it is smooth because we are at a generic point of the space time moduli space). Therefore, the topology of the classical space should be modified. Such a change of the topology of the moduli space between the classical and the quantum theory was first found in [14]. In this particular example it has already been established using other methods by Witten [15]. Classically the non-Abelian $S U(2)$ gauge symmetry is restored at the origin. However, in the quantum theory the origin is absent and the non-Abelian gauge symmetry is never restored. Similarly, the global $S U(2)_{R 2}$ is always broken [15].

$S U(2)$ with $N_{f}=1$. This theory is obtained on the 2-brane at a non-generic point in the space time moduli space. It occurs when classically one of the 6-branes coincides with the orientifold and the 2-brane is near that point. There is no enhanced continuous symmetry and no Higgs branch. As there is no enhanced continuous global symmetry on the brane and no enhanced continuous gauge symmetry in space time, the metric on K3 is not singular. Therefore, the moduli space of the three dimensional theory is smooth. Hence, the "W bosons" and the matter fields are never massless.

$S U(2)$ with $N_{f}>1$. This theory can be found when $N_{f} 6$-branes touch the orientifold and the 2-brane is near that point. At these points there is an enhanced $S O\left(2 N_{f}\right)$ global symmetry on the brane and an enhanced $S O\left(2 N_{f}\right)$ gauge symmetry in space time. The Higgs branch of that theory was analyzed in [9]. The global symmetry is broken as

$$
S O\left(2 N_{f}\right) \rightarrow \begin{cases}S U(2) & \text { for } N_{f}=2 \\ S O\left(2 N_{f}-4\right) \times S U(2) & \text { for } N_{f} \geq 3\end{cases}
$$

and the massless hypermultiplets transform like

$$
\begin{array}{cc}
\mathbf{1} & \text { for } N_{f}=2 \\
\frac{1}{2}\left(\mathbf{2 N}_{\mathbf{f}}-\mathbf{4}, \mathbf{2}\right) \oplus(\mathbf{1}, \mathbf{1}) & \text { for } N_{f} \geq 3
\end{array}
$$

where the $\frac{1}{2}$ denotes half hypermultiplets in that representation. The quantum theory is asymptotically free for any $N_{f}$. Far from the singularity the moduli space asymptotes to the one loop corrected answer but unlike the previous case the singularity is not smoothed out. From the enhanced $S O\left(2 N_{f}\right)$ gauge symmetry in space time we know that the K3 has a $D_{N_{f}}$ singularity. Therefore, the moduli space of the three dimensional gauge theory also 
has such a singularity. As in [13] we interpret the Higgs branch as the moduli space of a space time instanton. This interpretation is consistent with (3) and (4) and with the fact that $S O(32)$ instantons were interpreted in [10] as the Higgs branch of the D5-brane which is our probe. As in the $U(1)$ theory with $N_{f}>1$ flavors, the theory at the singularity must be in a non-trivial fixed point of the renormalization group.

It is trivial to extend the discussion to study other gauge groups by using several coinciding 2-branes as a probe. Another, less trivial, extension of this work is to identify the three dimensional theories whose Coulomb branch has an $E$ type singularity. These will occur for special values of the space time moduli. The corresponding three dimensional theories have global $E_{6,7,8}$ symmetries. They are closely related to the still mysterious theories associated with small $E_{8}$ instantons [16,17].

It is easy to repeat this analysis in lower dimensions. Compactifying the type I theory on $T^{4}$ and wrapping the D5-brane on $T^{4}$ we find a string in six dimensions. It is identified with the elementary string of the dual type IIA theory compactified on K3. The moduli space of the theory on that brane in the classical approximation is $T^{4} / Z_{2}$ [10]. The interpretation of this paper is that this space becomes a K3 (which is smooth for generic moduli) by the quantum effects on the two dimensional world sheet of the brane (or by some space time effect such that it is consistent with the two dimensional dynamics). One can straightforwardly extend this discussion to compactifications to five and even four dimensions.

\section{Acknowledgements}

This work was supported in part by DOE grant DE-FG02-96ER40559. We thank T. Banks, M. Douglas, D. Morrison, A. Sen, S. Shenker and especially E. Witten for helpful discussions. 


\section{References}

[1] For a nice review see, S. Chaudhuri, C. Johnson, and J. Polchinski, "Notes on DBranes," hep-th/9602052.

[2] U.H. Danielsson, G. Ferretti and B. Sundbor, "D-particle Dynamics and Bound States," hep-th/9603081.

[3] D. Kabat and P. Pouliot, "A Comment on Zero-brane Quantum Mechanics," hepth/9603127

[4] M. R. Douglas, "Gauge Fields and D-branes," hep-th/9604198.

[5] T. Banks, M.R. Douglas and N. Seiberg, "Probing F-theory With Branes," hepth/9605199

[6] M. R. Douglas, D. Kabat, P. Pouliot and S. H. Shenker, to appear.

[7] A. Sen, "F-theory and Orientifolds," hep-th/9605150.

[8] C. Vafa, "Evidence for F-theory," hep-th/9602022.

[9] N. Seiberg and E. Witten, Nucl. Phys. BB431 (1994) 484, hep-th/9408099.

[10] E. Witten, "Small Instantons in String Theory," hep-th/9511030, Nucl. Phys. B460 (1995) 541.

[11] E. Witten, "String Theory Dynamics in Various Dimensions," hep-th/9503124, Nucl. Phys. B443 (1995) 85.

[12] P.K. Townsend, "String-Membrane Duality in Seven Dimensions," hep-th/9504095, Phys. Lett. 354B (1995) 247.

[13] M. R. Douglas, "Branes within Branes," hep-th/9512077.

[14] N. Seiberg, "Exact Results on the Space of Vacua of Four Dimensional SUSY Gauge Theories," hep-th/9402044, Phys. Rev. D49 (1994) 6857.

[15] E. Witten, unpublished.

[16] O. Ganor and A. Hanany, "Small E(8) Instantons and Tensionless Noncritical Strings," hep-th/9602120.

[17] N. Seiberg and E. Witten, "Comments on String Dynamics in Six Dimensions," hepth/9603003. 